Kallioniemi, Anne

\section{High-resolution CGH analysis on CDNA microarrays for direct identification of amplification target genes}

\author{
Elizabeth Hyman, Outi Monni, Ester Rozenblum, \\ Spyro Mousses, Michael Bittner, Yidong Chen, \\ Olli Kallioniemi \& Anne Kallioniemi
}

Cancer Genetics Branch, National Human Genome Research Institute, National Institutes of Health, Bethesda, Maryland, USA

We sought to identify amplification target genes across the genome on the basis of a modification of the protocol developed by Pollack et al. ${ }^{1}$ for comparative genomic hybridization (CGH) analysis on complementary DNA microarrays. The same cDNA microarrays are then applied for expression analyses to identify directly genes whose expression is increased owing to amplification. We tested this approach by analyzing five breast cancer cell lines using a custom-made cDNA microarray specific for chromosome 17. The results were compared with data from conventional CGH analysis and indicated accurate detection of cell lines with HER-2 amplification (BT-474, SK-BR-3 and UACC-812) and those with amplification of the 17q23 region (BT-474 and MCF7). Compared with the exact copy number values available by fluorescence in situ hybridization, the CGH microarray analysis accurately defined the location and detailed structure of the amplicons along 17q23. Although we observed individual cDNA clones providing false-negative copy number estimates, averaging out signals over multiple adjacent clones provided accurate definition of all high-level amplifications. We observed numerous specific genes that showed both amplification and overexpression, such as HER-2, GRB7, MLN51 and MLN64 at17q12 as well as $S 6 \mathrm{~K}$ and $A P P B P 2$ at $17 \mathrm{q} 23$. A few microarray experiments carried out within a two-week period allowed us to reproduce and extend the information obtained by conventional techniques over the course of the past few years. We are now carrying out CGH microarray analyses of these and other cell lines with an 8,000-clone array, which provides coverage of the entire genome at once.

1. Pollack, J.R. Nature Genet. 23, 41-46 (1999).

Kaminski, Naftali [23]

\section{A composite genomic profile of lung cancer in smokers and nonsmokers}

Naftali Kaminski1,2, David Pilzer ${ }^{3}$, Gady Cojocaru1 ${ }^{4}$, Ofer Margalit ${ }^{1,4}$, Nir Friedman ${ }^{5}$, Shlomit Reinstein ${ }^{3}$, David Simansky ${ }^{6}$, Penina Yaron ${ }^{2}$, Amariglio Ninnete ${ }^{4}$, Alon Yellin ${ }^{6}$, Gideon Rechavi ${ }^{4}$, Eitan Friedman ${ }^{3}$ $\&$ Meir Krupsky²

${ }^{1}$ Department of Functional Genomics, Sheba Medical Center, Tel Hashomer, Israel ${ }^{2}$ Institute of Respiratory Medicine, Sheba Medical Center, Tel Hashomer, Israel ${ }^{3}$ Susanne Levy Gertner Oncogenetics Unit, Sheba Medical Center, Tel Hashomer, Israel

${ }^{4}$ Department of Pediatric Hemto-oncology, Sheba Medical Center, Tel Hashomer, Israel

${ }^{5}$ Department of Computer Sciences, Hebrew University of Jerusalem, Jerusalem, Israel

${ }^{6}$ Department of Thoracic Surgery, Sheba Medical Center, Tel Hashomer, Israel

The molecular mechanisms that lead to lung cancer in smokers and nonsmokers are largely unknown. This study created a composite genomic profile of the most common type of lung cancer, non-small-cell lung cancer, in smokers and non- smokers using comparative genomic hybridization and oligonucleotide microarrays. Comparative genomic hybridization on 24 tumor specimens from 14 smokers and 10 nonsmokers with resectable (I-IIIa) non-small-cell lung cancer revealed an abnormal chromosomal copy number in 21 of 24 tumors. The number of changes in smokers was significantly higher than that in nonsmokers $(P=0.005)$. Amplifications of chromosomes $2(7 / 14 ; 50 \%)$ and $4(5 / 14 ; 35.7 \%)$ were detected exclusively in smokers, whereas amplifications of chromosome 5 and allelic losses involving chromosomes 19 and 22 were equally distributed. In subsequent analyses we used Affymetrix GeneChip U95A microarrays, which allow for the analysis of approximately 11,000 full-length human genes, to analyze the gene expression patterns that correlate with these genomic changes as well as to provide a comprehensive transcriptional profile of non-small-cell lung cancer in smokers and nonsmokers. Preliminary analysis of the data revealed distinct gene expression patterns that correlated with the genomic changes. We believe that our approach, combining analysis of genomic content and alteration with analysis of gene expression, will lead to a composite genomic profile of cancer in smokers and nonsmokers, resulting in better tools for early detection, improved prediction of prognosis and discovery of new drugs to treat this devastating disease.

Kannan, Karuppiah

[24]

\section{Primary and secondary target genes regulated by $\mathrm{p} 53$ identified by DNA microarrays}

Karuppiah Kannan ${ }^{1}$, Ninette Amariglio ${ }^{2}$, Gideon Rechavi ${ }^{2}$, Yasmin Yaakov², Naftali Kaminski², Gad Getz ${ }^{1}$,

Eitan Domany ${ }^{1} \&$ David Givol ${ }^{1}$

${ }^{1}$ Weizmann Institute of Science, Rehovot, Israel

${ }^{2}$ Chaim Sheba Medical Center, Tel Hashomer, Israel

The function of p53 as a tumor suppressor is mainly due to its activity as a transcription factor, activating many genes in response to genotoxic stress. This activity may be the basis for its ability to protect cells against DNA damage leading to growth arrest or apoptosis. The profile of gene expression induced by p53 is heterogeneous, varying across cell lines and tissues, and it is likely that some of the $\mathrm{p} 53$-induced genes may be secondary to its primary role as a transcription factor. The principal targets for p53 may themselves be of great value as tumor inhibitors, as was demonstrated for p21waf and MDM2. In order to distinguish between primary and secondary targets for p 53 regulation, we analyzed the profile of gene expression regulated by $\mathrm{p} 53$ in the presence and absence of inhibitors of protein synthesis, using oligonucleotide microarrays containing probes for approximately 7,000 human genes. Only $25 \%$ of the p53-induced genes are also induced in the absence of protein synthesis and may be considered primary targets. We used cluster analysis of these data to classify the p53-regulated genes into early and late genes according to the kinetics of their expression. The results indicate that p53 simultaneously regulates a plethora of genes involved in many cellular functions, such as DNA repair, cytoskeleton and extracellular matrix repair, signal transduction and oxidoreduction as well as growth arrest and apoptosis. 